\title{
Binding specificity of ASHH2 CW-domain towards H3K4me1 ligand is coupled to its structural stability through its $\alpha 1$-helix
}

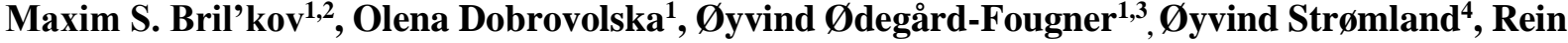 \\ Aasland $^{* 5}$, Øyvind Halskau*1
}

${ }^{1}$ Department of Biological Sciences, University of Bergen, N-5020 Bergen, Norway.

${ }^{2}$ Department of Pharmacy, University of Troms $\varnothing$. N-9037, Troms $\varnothing$, Norway.

${ }^{3}$ Department of Molecular Cell Biology, Institute for Cancer Research, The Norwegian Radium Hospital, N-0379 Oslo, Norway.

${ }^{4}$ Department of Biomedicine, University of Bergen, N-5020, Bergen, Norway.

${ }^{5}$ Department of Biosciences, University of Oslo, N-0316 Oslo, Norway.

* Correspondence regarding the molecular biology of histone binding domains should be addressed to Rein Aasland, e-mail: rein.aasland@ibv.uio.no. Correspondence regarding structure and biophysics should be addressed to Øyvind Halskau, e-mail: oyvind.halskau@uib.no.

\section{Abstract:}

The CW-domain binds to histone-tail modifications found in different protein families involved in epigenetic regulation and chromatin remodelling. $\mathrm{CW}$-domains recognize the methylation state of the fourth lysine on histone 3 , and could therefore be viewed as a reader of epigentic information. The specificity towards different methylation states such as me1, me 2 or me 3 depends on the particular CW subtype. For example, the $\mathrm{CW}$ domain of ASHH2-methyltransferase binds preferentially to H3K4me1, MORC3 binds to both H3K4me2 and me3 modifications, while ZCWPW1 is more specific to H3K4me3. The structural basis for these preferential bindings are not well understood, and recent research suggests that a more complete picture will emerge if dynamical and energetic assessments are included in the analysis of interactions. This study uses fold assessment by NMR in combination with mutagenesis, ITC affinity measurements and thermal denaturation studies to investigate possible couplings between ASHH2 CW selectivity towards $\mathrm{H} 3 \mathrm{~K} 4 \mathrm{me} 1$ and the stabilization of the domain and loops implicated in binding. Key elements of the binding site - the two tryptophans and the $\alpha 1$-helix form and maintain the binding pocket were perturbed by mutagenesis and investigated. Results show that $\alpha 1$-helix maintains the overall stability of the fold via the I915 and L919 residues, and that correct binding consolidates the loops designated $\eta 1, \eta 3$, as well as the C-terminal. This consolidation is incomplete for $\mathrm{H} 3 \mathrm{~K} 4 \mathrm{me} 3$ binding to $\mathrm{CW}$, which experiences a decrease in overall thermal stability upon binding. Moreover, loop-mutations not directly involved in the binding site nonetheless affect the equillibrium positions of key residues.

Keywords:

Zinc-finger, histone methylation, histone modification reader, chromatin modification, proteinprotein interaction, SDG8, EFS, SET8 


\section{Abbreviations:}

PTM, post-translational modification; H3K4meX, histone tail peptide methylated 0-3 times at position Lysine 4; H3K36me3, Histone H3 trimethylation at the Lysine 36; hetNOE, heteronuclear nuclear Overhauser effect; ITC, isothermal titration calorimetry; NMR, nuclear magnetic resonance; NOE, nuclear Overhauser effect; HSQC, heteronuclear single quantum coherence; CSP, chemical shift perturbation.

\section{Introduction}

Regulation of gene expression and activity at the chromatin level relies on proteins that "read", "write" or "erase" post-translational modifications (PTMs) on histone tails and DNA. Proteins containing such domains are involved in genome organization and regulation by recognizing and modifying the PTM state of the genome compartments [1-3]. A key feature of histone tail recognition domains is that they can both recognize the general PTM state of their ligand while still have the ability to differentiate between them [4-6]. The CW domain family is a histone-tail methylation-state reader shared among numerous organisms (vertebrates, vertebrate-infecting parasites and higher plants). The name of the domain comes from its four conserved cysteines and three conserved tryptophans (Fig. 1A). The conserved cysteines coordinate a $\mathrm{Zn}^{2+}$ ion essential for folding, and two of the three tryptophan residues form a $\pi$-cation-based binding pocket with high affinity towards methylated lysine residues found on histone tails. The final tryptophan forms part of the hydrophobic core of the domain [7-9]. The $\mathrm{CW}$ domain appears within a multidomain functional context that varies from protein family to protein family [7]. One example of this is the MORC family of ATPase chromatin remodelers. Here, the CW domain recruits the proteins to the chromatin by recognizing $\mathrm{H} 3 \mathrm{~K} 4 \mathrm{me} 2 / 3$ modifications. $\mathrm{CW}$ also regulates the ATPase activity of MORC3 by suspending its autoinhibition after binding to methylated H3 histone tails [10-12]. Intrestingly, the MORC4 protein, while having hight structural and sequence similarity to MORC3, is not a autoinhibiting enzyme. For MORC4 activation, $\mathrm{CW}$ needs to interact with DNA in addition to binding H3K4me3 [13]. Another example are the ZCWPW1 and ZCWPW2 PWWPdomain-containing proteins. Within these proteins, the $\mathrm{CW}$ function is unclear, but they recognize H4K20 methylation marks in addition to the H3K4me3 specificity conferred by the CW domain $[8,14,15]$. In the transcriptional corepressor LSD2/AOF1/KDM1B that demethylates mono- and dimethyl H3K4 marks, CW appears to be inactive due to steric inaccessibility. However, the CW contributes to the overall structural stability of the protein and regulates the enzyme's activity and association with mitotic chromosomes [15-18]. In the MBD protein family, the ZmMBD101 protein maintains the repressed state of the Mutator genes, protecting plant genomes from mutagenesis caused by transposons. The role of the CW domain in this context is still unclear [19]. CW also appears in ASHH2 (other names are SDG8 and EFS), a methyltransferase found in the small, flowering plant Arabidopsis thaliana. ASHH2 is involved in the regulation of gene expression by the histone $\mathrm{H} 3$ trimethylation at Lys-36 (H3K36me3). Within ASHH2, the CW domain preferrentially binds the 
H3K4me1 mark and presumably helps docking the catalytic SET domain correctly onto the histone [9,20-22].

The CW domain paralogs have different affinities towards different methylation states of H3K4. While the ASHH2 CW domain has higher affinity towards H3K4me1 $[9,23]$, the rest of the known CW domains bind stronger to $\mathrm{H} 3 \mathrm{~K} 4 \mathrm{me} 2$ and $\mathrm{H} 3 \mathrm{~K} 4 \mathrm{me} 3$ modifications [8,10,11,15]. Earlier, factors determining the ligand methylation state specificity of $\mathrm{CW}$ domains have been presented and discussed $[9,23,24]$. The C-terminal regions of the CW domain due to its variability between paralogs were suggested to be involved in ligand specificity. For example, the CW-domain of ZCWPW1 is unique in that it has a non-conserved tryptophan residue (Trp303) at its C-terminal end (Fig. 1B), and this tryptophan finalizes the binding pocket upon binding. Mutation of this tryptophan leads to reduced affinity [8]. Its homolog ZCWPW2 has a phenylalanine residue (Phe78) in this position which also completes the binding pocket and might contribute to selectivity of the methylation state (Fig. 1B) [15]. The CW domain of MORC3 proteins, in contrast, has Glu453 residue (Fig. 1B), which finalizes the binding pocket and facilitates binding to methylated H3K4 peptides. Despite having the highest affinity towards $\mathrm{H} 3 \mathrm{~K} 4 \mathrm{me} 3$ modification, its ability to differentiate between methylation states is reduced relative to what is observed for other $\mathrm{CW}$ domains $[10,11,15]$. The ASHH2-subtype possesses a unique C-terminal $\alpha 1$-helix located right above the tryptophan binding pocket (Fig. 1B). Although not conserved, this helix is also part of the binding pocket $[23,24]$, as removal of this element leads to loss of ligand binding ability [9].

Recent X-ray (Liu et al., PDB code: 5YVX) ) and NMR (Dobrovolska et al., PDB code: 6QXZ) structural studies described the $\mathrm{CW}$ of ASHH2 in complex with $\mathrm{H} 3 \mathrm{~K} 4 \mathrm{me} 1$. The published structures agreed on the core of the complex; however, the structural data related to the $\alpha 1$-helix and the following C-terminal regions differ [23,24]. The studies also concluded differently with respect to binding mechanisms and determinants. The key residues for binding proposed by Liu et al. were L915, N916 and I919, residing on the $\alpha 1$-helix. These were proposed based on the crystal structure of the CWH3K4me1 complex, where the domain construct ended right after the $\alpha 1$-helix (residue I921) and contained a mutation necessary for crystallization (E917A) close to the residues they identify as crucial for binding (Fig. 1A). Dobrovolska et al. argued that the N916 residue is not a part of the interaction mechanism, as the NMR structure of the complex showed that this residue does not make any contacts with the ligand. The studies agreed, however, that L915 and I919 locked the methylated lysine of the ligand inside the binding pocket. The construct used by Dobrovolska et al. to solve the structure was longer at the C-terminal than the one used in the Liu et al. study, which allowed elucidation of the role of the I921-Q923 region in binding. Analysis of the domain's dynamics and flexibility using MD simulations and NMR further indicated that the binding mode of $\mathrm{CW}$ is described best by a conformational selection model [24]. A conformational selection mechanism requires that the protein has a fluctuating structural ensemble that includes the conformation(s) required for binding, even in the 

its structural stability through its $\alpha 1$-helix

absence of ligand $[25,26]$. In such scenarios, any point mutations could potentially perturb the domain's fluctuating fold, distorting the interaction mechanism that depends on fine-tuned equilibriums.

The objective of the work was to investigate the possible coupling between selectivity determinants and protein fold stability that allows ASHH2 $\mathrm{CW}$-domain to preferentially recognize H3K4me1. In order to achieve this goal, we prepared two mutants affecting the positioning of the tryptophans of the binding site and two mutants involved in positioning the $\alpha 1$-helix in the ligand binding site. To assess the fundamental importance of this helix, we also prepared a deletion mutant, removing it entirely. We determined the affinities of $\mathrm{H} 3 \mathrm{~K} 4 \mathrm{me} 1 / 2 / 3$ interacting with these mutants using ITC, performed thermal stability studies in the presence and absence of histone $\mathrm{H} 3$ mimicking peptides, and assessed changes to their fold using NMR fingerprinting.

\section{Results}

\section{ASHH2 CW-domain interaction with $\mathrm{H} 3$ mimicking peptides}

Fold assessment and chemical shift perturbation analysis

The ASHH2 CW-domain binds $\mathrm{H} 3 \mathrm{~K} 4 \mathrm{me} 1, \mathrm{H} 3 \mathrm{~K} 4 \mathrm{me} 2$ and $\mathrm{H} 3 \mathrm{~K} 4 \mathrm{me} 3$, with the highest affinity towards H3K4me1 [9]. It is not known, however, whether the binding of these peptides is essentially the same or whether they affect the CW's fold differently. ${ }^{1} \mathrm{H}^{-15} \mathrm{~N}$ HSQC NMR spectra of CW in complex with mono-, di-, and trimethylated ligand were acquired and compared to the unbound form. Using the backbone chemical shift assignments available for the four situations [27], the chemical shift perturbation (CSP) was calculated, and the residues involved in the corresponding complex formation were determined (Fig. 2A). The NMR data in the case of all the three histone-mimicking peptides suggests that binding causes fairly extensive structural changes that are not limited to W865 and W874 of the binding pocket. However, the HSQC spectra confirms that the CW-domain remains folded when complexed with each of the peptides (Fig. S1). This observation is in line with the flexible nature of ASHH2 CW and the proposed mechanism of conformational selection [24]. The most notable changes in chemical shifts are found in the first $\beta$-strand, perhaps related to the nascent $\beta$-sheet augmentation discussed in [24], in the conserved W891 that is not part of the binding pocket, in the a1-helix and the १1-loop (Fig. 2A).

The CSP data for the H3K4me1 peptide shows that E913 in the a1-helix and I921-Q923 in the C-terminal tail are involved in the binding. Earlier, it was shown that I921 and Q923 are two of the key residues establishing contacts with $\mathrm{H} 3 \mathrm{~K} 4 \mathrm{me} 1$ and forming part of the final cage-like configuration around $\mathrm{K} 4 \mathrm{me} 1$ [24]. In the case of the interaction with $\mathrm{H} 3 \mathrm{~K} 4 \mathrm{me} 2$ and $\mathrm{H} 3 \mathrm{~K} 4 \mathrm{me} 3$, the CSP values for these residues are less prominent, suggesting that they might not only be involved in mediating the interaction with the ligand but also contribute to selectivity towards $\mathrm{H} 3 \mathrm{~K} 4 \mathrm{me} 1$. Binding to the $\mathrm{H} 3 \mathrm{~K} 4 \mathrm{me} 2$ peptide has its most significant effect on W865 in the binding pocket, L873, the 11 -region, M910, N916 and L919 of the $\alpha 1$-helix. Binding to H3K4me3 affected the structure to a somewhat larger degree, as 

its structural stability through its $\alpha 1$-helix

the combined chemical shift values are slightly higher for the majority of the amino acids, especially at $\eta 2$ and $\eta 3$-loops (Fig. 2A). Yet, overall, the CSP data do not show much difference in how the backbone was affected by the three different peptides. The perturbation analysis takes into account only the backbone amide correlations, which may be insufficient for detection of all features of these interactions.

Amino acid side chain signals, corresponding to the NH groups of the tryptophans and asparagines and not included in the CSP analysis above, experience large shifts as a result of ligand binding. Notably, in each case of the ligand methylation state, the same NH signals had different shifts, indicating that the chemical environment around these groups was affected differently (Fig. 2B). The shifts suggest a change in the configuration of the tryptophan side-chains in the binding pocket to accommodate the additional methyl group(s) of the ligand. A similar observation for the signals corresponding to the side-chains of N912 and N916 suggests that the $\alpha 1$-helix also experience methylation-dependent shifts in their positions (Fig. 2C and 2D).

\section{Dynamic properties of $\mathrm{CW}$ domain bound to histone mimicking peptides}

The HSQC fingerprinting, CSP analysis and differences in the chemical shifts of the side chain of the tryptophans and asparagines together suggest there may be subtle differences in how the ASHH2 $\mathrm{CW}$ domain responds to different ligand methylation states. Such differences may not be readily detectable by assessing chemical shifts only, as some they reside among fluctuations in the domain's structure and its dynamic properties that contribute little to crosspeak positions. Dobrovolska et al. provided a full NMR dynamics characterization for CW in the unbound and H3K4me1-bound state [24]. Here we expand part of this analysis by accumulating and comparing the hetNOE data for H3K4me1/2/3 peptides and compare them.

Overall, the average hetNOE values for the domain bound to the peptides were around 0.9, indicating moderate flexibility, except in the $\eta 1$-loop, which remained quite flexible with hetNOE values as low as 0.6-0.8 (Fig. 3). Generally, the binding of the H3K4me1 peptide results in movement restriction of the unstructured C-terminal I921-Q923 region. The $\eta 3$-loop residue S907 shows high flexibility, as was reported in [24], with stabilization when CW-domain is complexed with H3K4me1 and $\mathrm{H} 3 \mathrm{~K} 4 \mathrm{me} 3$. Binding to the $\mathrm{H} 3 \mathrm{~K} 4 \mathrm{me} 2$ peptide also had a stabilizing effect on $\mathrm{S} 907$, but less pronounced. When bound to the H3K4me1 peptide, a few notable outliers appear, R876, S880, A902 and C904, which displayed hetNOE values higher than 1 (Fig. 3A). R876 is located on the $\beta 2$, S880 is located in the small helical feature which follows the $\beta 2$ strand, and A902 is located in another helical feature, formed upon complexation with $\mathrm{H} 3 \mathrm{~K} 4 \mathrm{me} 1$. The C904 residue is involved in the orientation of the $\mathrm{Zn}^{2+}$ ion in the core of the domain. The stability of the $\alpha 1$-helix region is slightly decreased for CW bound to H3K4me3 (Fig. 3C), which might have an effect on the complex stability. In sum, when complexed with me 2 and me 3 peptides, the dynamic behavior of $\mathrm{CW}$ looks more similar to the domain in its unbound state (Fig. 3B and C). This may indicate that the full shift in fold equlibrium occurs only with monomethylated ligand. 

its structural stability through its $\alpha 1$-helix

\section{Thermodynamical characterization of the interaction}

The NMR analysis suggests that CW, when complexed with the peptides, sample available conformations differently at equilibrium. If a given methylation state fail to stabilize the complex, this should be reflected in its thermal stabilities. The CW-domain in its unbound and bound state was therefore subjected to thermal denaturation analysis monitored by intrinsic tryptophan fluorescence. Complexation with $\mathrm{H} 3 \mathrm{~K} 4 \mathrm{me} 1$ and $\mathrm{H} 3 \mathrm{~K} 4 \mathrm{me} 2$ peptides increased the thermal stability of the domain: $\mathrm{T}_{\mathrm{m}}$ $=67.3{ }^{\circ} \mathrm{C}$ without peptide, $\mathrm{T}_{\mathrm{m}}=71.4{ }^{\circ} \mathrm{C}$ with $\mathrm{H} 3 \mathrm{~K} 4 \mathrm{me} 1$ and $\mathrm{T}_{\mathrm{m}}=71.6{ }^{\circ} \mathrm{C}$ with $\mathrm{H} 3 \mathrm{~K} 4 \mathrm{me} 2$. In contrast, interaction with $\mathrm{H} 3 \mathrm{~K} 4 \mathrm{me} 3$ reduced this stability to $\mathrm{T}_{\mathrm{m}}=64.9^{\circ} \mathrm{C}$ (Fig. $4 \mathrm{~A}$ and $\mathrm{B}$ ).

In order to study thermodynamical forces underlying ligand binding and selectivity, we performed ITC measurements of $\mathrm{CW}$ interacting with $\mathrm{H} 3 \mathrm{~K} 4 \mathrm{me} 1 / 2 / 3$ peptides. Interaction with $\mathrm{H} 3 \mathrm{~K} 4 \mathrm{me} 1$ peptide resulted in $\mathrm{K}_{\mathrm{d}}=1.31 \pm 0.32 \mu \mathrm{M}$, binding enthalpy $\Delta \mathrm{H}=-89.25 \pm 8.58 \mathrm{~kJ} / \mathrm{mol}$ and entropy $\Delta \mathrm{S}=-192.42 \pm 15.31 \mathrm{~J} / \mathrm{mol} \cdot \mathrm{K}$. Interaction with $\mathrm{H} 3 \mathrm{~K} 4 \mathrm{me} 2$ resulted in $\mathrm{K}_{\mathrm{d}}=4.61 \pm 0.28 \mu \mathrm{M}$, binding enthalpy to $\Delta \mathrm{H}=-83.64 \pm 3.11 \mathrm{~kJ} / \mathrm{mol}$ and entropy to $\Delta \mathrm{S}=-178.37 \pm 10.82 \mathrm{~J} / \mathrm{mol} \cdot \mathrm{K}$. Interaction with $\mathrm{H} 3 \mathrm{~K} 4 \mathrm{me} 3$ displayed weaker binding relative to both $\mathrm{H} 3 \mathrm{~K} 4 \mathrm{me} / 3$, with a dissociation constant $\mathrm{K}_{\mathrm{d}}=14.18 \pm 0.74 \mu \mathrm{M}$. Corresponding binding enthalpy and entropy were $-60.14 \pm 1.38 \mathrm{~kJ} / \mathrm{mol}$ and $-108.90 \pm 5.00 \mathrm{~J} / \mathrm{mol} \cdot \mathrm{K}$, respectively. Stoichiometric coefficients were in the range of $0.82-1.08$. As a control, the unmodified H3K4 peptide was used and showed no binding. Results of the analysis are summarized in Fig. 5 and Table S3, representative isotherms are shown in supplementary Fig. S2.

\section{Effect of $\alpha 1$-helix mutations on binding affinity, domain conformation and thermal stability}

A detailed structural study of $\mathrm{CW}$ binding to H3K4me1 showed that the $\alpha 1$-helix and the disordered region following it contribute critically to binding. A group of $\mathrm{CW}$ residues residing in this region establish direct NOE contacts with the ligand and comprise L919, I915 and Q923 [24]. Hoppmann et al. showed that without this part, the protein is no longer capable of binding a ligand. It is, however, unclear whether this is due to loss of fold, or inability to retain the ligand in the otherwise intact binding site. To assess the impact $\alpha 1$-helix has on the fold of the protein, the CW $\Delta$ LID mutant (residues 847-910 removed) was generated, and a ${ }^{1} \mathrm{H}$ NMR spectrum was acquired. Compared to the wild type, the spectrum of the CW $\Delta$ LID shows fewer and broader peaks spread across a narrower region, which confirms that removal of the $\alpha 1$-helix leads to loss of fold (Fig. 6A). This finding confirms the joint importance of both ligand binding and fold maintainance of the $\alpha 1$-helix and the C-terminal region.

To examine the role of the $\alpha 1$-helix in domain stability and specificity, we prepared I915A and L919A mutants. Their ${ }^{1} \mathrm{H}-{ }^{15} \mathrm{~N}$ HSQC NMR spectra showed that the protein remains folded (Fig. 6D and E). Compared to the wild type, I915A and L919A mutations show animpact on the overall fingerprint, as more than $60 \%$ of the peaks were shifted substantially (more than $0.2 \mathrm{ppm}$ ) (Fig. 6B). Thermal denaturation experiments showed that compared to the native $\mathrm{CW}$ domain $\left(\mathrm{T}_{\mathrm{m}}=67.3^{\circ} \mathrm{C}\right)$, the L919A mutation significantly decreased the stability of the protein by $10.4{ }^{\circ} \mathrm{C}\left(\mathrm{T}_{\mathrm{m}}=56.9^{\circ} \mathrm{C}\right)(\mathrm{Fig} .4 \mathrm{C}$ and D). Moreover, the binding of $\mathrm{H} 3 \mathrm{~K} 4 \mathrm{me} 1$ decreased it further, by more than 10 degrees in addition 
$\left(\mathrm{T}_{\mathrm{m}}=46.7^{\circ} \mathrm{C}\right.$ ). This behaviour strikingly contrasts with the wild-type where the ligand increases the $\mathrm{T}_{\mathrm{m}}$ by $\approx 5{ }^{\circ} \mathrm{C}$. 1915 mutation had a more dramatic impact on the fold stability, as it was not possible to fit a curve to the data from denaturation analysis, as the data does not display a sigmoidal model.

Changes in the binding preference for I915A mutant was previously evaluated using ITC by Liu et al., with the conclusion linking it to a change in specificity from $\mathrm{H} 3 \mathrm{~K} 4 \mathrm{me} 1$ to $\mathrm{H} 3 \mathrm{~K} 4 \mathrm{me}$. When we performed the ITC experiment with the I915A mutant using our slightly longer construct (CW42), it was not possible to obtain an accurate $K_{d}$ and other thermodynamic parameters, presumably due to its weak interaction with the peptides. The interaction of L919A mutant with H3K4me2 and H3K4me3 peptides could not be characterized either. In the case of H3K4me1 interaction with L919A, the $K_{d}$, the binding enthalpy, and entropy were determined to be $12.17 \pm 2.10 \mu \mathrm{M},-76.92 \pm 2.31 \mathrm{~kJ} / \mathrm{mol}(\Delta \mathrm{H})$, and $-163.8 \pm 6.61 \mathrm{~J} / \mathrm{mol} \cdot \mathrm{K}(\Delta \mathrm{S})$, respectively. The stoichiometric coefficients were in the range of $0.86-$ 0.88. The results are summarized in Fig. 5 and Table S3. The shift in specificity reported by Liu et al. was not confirmed in our experiments [23].

\section{Binding pocket flexibility as possible determinants for binding preferences}

Binding mode and ligand specificity of each CW subtype must ultimately arise from the environment provided by the binding pockets. To compare structures, we performed sequence and structural alignments of CW domains from ASHH2 (2L7P), MORC3 (5SVX), and ZCWPW1 (2E61) (Fig. 1A). Structural superimposition of ZCWPW1, ZCWPW2, MORC3 (human and mouse), and ASHH2 CW revealed different angles between the tryptophans side-chains in the binding pocket (Fig. 1D). ASHH2 has the narrowest angle $\left(65^{\circ}\right)$, which might partially explain its specificity towards $\mathrm{H} 3 \mathrm{~K} 4 \mathrm{me} 1$. In the remaining structures, with a reported preference towards $\mathrm{H} 3 \mathrm{~K} 4 \mathrm{me} 3$, the angle varies between $77^{\circ}$ and $113^{\circ}$. Upon ligand binding, the angle increases, widening the binding pocket (results are summarized in Table 1). This expansion might be possible due to the flexibility of the loops surrounding the binding pocket, with ASHH2 having insufficient flexibility to open up the pocket for stable binding to the H3K4me3 ligand. The gap in ASHH2 and MORC3 sequence alignments (Fig. 1A) translates into a shorter loop between two $\beta$-sheets, compared to ZCWPW1 (Fig. 1D). Inside this loop, MORC3 has alanine in the place of ASHH2's D870, and ZCWPW1 has the sequence S867-FP-N870 inserted relative to ASHH2. These variations in length and composition of the loop may impact the orientation of the $\beta$-strands and thus on also the positioning of the conserved tryptophans in the binding pocket.

Loops have been implicated in the binding mechanism of the $\mathrm{CW}$ domain, where they regulate the positioning of $\alpha 1$-helix ( $\eta 3$-loop and the post-helix C-terminal coil). Additionally, the $\eta 1$-loop also interact with the ligand upon complex formation, and together these unstructured elements mediate binding and stabilize the complex [24]. To study the role of the loop that connects the two $\beta$-sheets scaffolding the binding pocket, the D870A and insC866-SFPN-C871 mutants were prepared, with mutations that correspond to the composition of MORC3 and ZCWPW1 loops respectivelly. The folded 

its structural stability through its $\alpha 1$-helix

states of the mutants were verified by ${ }^{15} \mathrm{~N}$ HSQC fingerprinting (Fig. 6B). The D870A mutant had little effect on the structure, affecting only around 7\% of the signals in the fingerprint. The insC866-SFPNC871 insertion mutant, had a more pronounced effect on the structure, resulting in a shift of around $45 \%$ of signals (Fig. 6B). Changes in the binding preferences of the D870A and insC866-SFPN-C871 mutants were evaluated by ITC.

It was not possible to obtain $\mathrm{K}_{\mathrm{d}}$ and other thermodynamic parameters for the insC866-SFPNC871 mutant due to weak interactions with all three histone mimicking peptides. Change in selectivity was not observed, and interaction of the D870A sample with H3K4me1 was comparable to WT values $\left(\mathrm{K}_{\mathrm{d}}=1.37 \pm 0.32 \mu \mathrm{M}\right.$, binding enthalpy $\Delta \mathrm{H}=-79.22 \pm 4.81 \mathrm{~kJ} / \mathrm{mol}$ and entropy $\Delta \mathrm{S}=-153.27 \pm 13.75$ $\mathrm{J} / \mathrm{mol} \cdot \mathrm{K})$. This mutation gave decreased binding affinity towards $\mathrm{H} 3 \mathrm{~K} 4 \mathrm{me} 2$ and me 3 peptides, however: interaction with $\mathrm{H} 3 \mathrm{~K} 4 \mathrm{me} 2$ resulted in $\mathrm{K}_{\mathrm{d}}=7.48 \pm 0.72 \mu \mathrm{M}, \Delta \mathrm{H}=-93.33 \pm 3.65 \mathrm{~kJ} / \mathrm{mol}$ and $\Delta \mathrm{S}=$ $-214.77 \pm 12.06 \mathrm{~J} / \mathrm{mol} \cdot \mathrm{K}$. For $\mathrm{H} 3 \mathrm{~K} 4 \mathrm{me} 3$, these binding parameters were $\mathrm{K}_{\mathrm{d}}=18.53 \pm 2.17 \mu \mathrm{M}, \Delta \mathrm{H}=$ $-57.47 \pm 2.37 \mathrm{~kJ} / \mathrm{mol}$, and $\Delta \mathrm{S}=-102.14 \pm 8.90 \mathrm{~J} / \mathrm{mol} \cdot \mathrm{K}$. The stoichiometric binding coefficients were in the range of 0.96 - 1.01. Results are summarized in Fig. 5 and Table S3 with representative isotherms shown in supplementary Fig. S2.

\section{Effect of mutations on the environment of the tryptophan side chains}

Chemical shift analysis of the unbound state and of the H3K4me1-3 bound situations suggested that side chains were more sensitive to the binding pocket environment compared to their respective backbone atoms. Therefore, we also compared the spectra of insC866-SFPN-C871, D870A, and I915A, L919A to the spectra of the WT with a focus on sidechain NH crosspeaks. Signals from the $\varepsilon$-NH nuclei of the aromatic side chain of tryptophans are located in the ${ }^{1} \mathrm{H}$ 7-11 ppm and ${ }^{15} \mathrm{~N} 128-130 \mathrm{ppm}$ region of ${ }^{1} \mathrm{H}^{15} \mathrm{~N}$ HSQC spectra. Mutations in the $\alpha 1$-helix did not affect the core tryptophan W891 but significantly affected the tryptophan side chains in the binding pocket (W865 and W874). For L919A,

${ }^{15} \mathrm{~N} \varepsilon$ resonances were shifted upfield, maintaining a similar pattern as in the wild type domain. The I915A mutation affected the side chains somewhat differently, with the ${ }^{15} \mathrm{~N} \varepsilon$ resonance shifted downfield in the case of W865 (Fig. 6C). The mutant with the MORC3-like loop maintained a chemical environment similar to the WT situation, with slight shift of the signal from the core tryptophan side chain (W891). The mutant with the ZCWPW1-like loop had the most pronounced effect on the core tryptophan (W891), and also shifting the ${ }^{15} \mathrm{~N} \varepsilon$ resonances of binding pocket's tryptophan side chains upfield (W865 and W874) (Fig. 6C).

\section{Discussion}

Analysis and comparison of known of CW domains structures suggested that the specificity of the domain might be related to the geometry of the binding pocket, formed by tryptophan side chains, fluctuating conformers and flexible nature of the protein. Exploring the dynamic and energetic determinants of the ASHH2 CW-domain and several mutants, we found that the only in the case 

its structural stability through its $\alpha 1$-helix

interaction with $\mathrm{H} 3 \mathrm{~K} 4 \mathrm{me} 1$ did binding result in a fold-stabilization. We also found that the $\alpha 1$-helix was a requirement for the domain fold, and that mutations in loop structures had a marked effect on it thermal stability, fold and ability to interact with $\mathrm{H} 3 \mathrm{~K} 4 \mathrm{me} 1 / 2 / 3$ histone mimicks.

Early characterization of ASHH2 CW-domain behaviour was reported by Hoppmann et al., and expanded with the structures of the bound state by Liu et al. and Dobrovolska et al. In this study, the $\mathrm{K}_{\mathrm{d}}$ values for $\mathrm{CW}$ interaction obtained using ITC do not match entirely with the values obtained by Hoppmann et al. for H3K4me2 ( $\mathrm{K}_{\mathrm{d}}$ of $2.1 \mu \mathrm{M}$ vs. $4.61 \mu \mathrm{M}$ in the present study) and $\mathrm{H} 3 \mathrm{~K} 4 \mathrm{me} 3\left(\mathrm{~K}_{\mathrm{d}}\right.$ of $4 \mu \mathrm{M}$ vs. $14.18 \mu \mathrm{M}$ in the present study), but are comparable in the case of $\mathrm{H} 3 \mathrm{~K} 4 \mathrm{me} 1$. Comparing to Liu et al. data, the values reported are in better agreement. The discrepancy may be explained by differences in methods and protein constructs used. Hoppmann et al. employed SPR, which is sensitive to mass transfer, re-binding, and surface effects $[28,29]$. In addition, the study used a GST-ASHH2-CW construct, where the presence of the GST-tag could potentially affect the interaction [30]. However, both studies conclude that $\mathrm{H} 3 \mathrm{~K} 4 \mathrm{me} 1$ is the strongest binder, whereas $\mathrm{H} 3 \mathrm{~K} 4 \mathrm{me} 3$ is the weakest.

The ITC data shows that peptide binding is enthalpy-driven, and the difference in affinity arises from different enthalpy-entropy contributions [31,32]. H3K4me1 shows the most favourable enthalpy contribution of the three histone-mimicking peptides. Interaction with the other peptides showed a smaller enthalpy contribution. As the change in enthalpy in non-strict terms characterizes the number of non-covalent bonds formed upon complexation [33], this can be interpreted as the formation of fewer non-covalent interaction contacts for $\mathrm{H} 3 \mathrm{~K} 4 \mathrm{me} 2$ and -me 3 cases. Interaction with these peptides is also characterized by an increase in entropy terms when compared to H3K4me1 interaction. This is consistent with a higher degree of mobility of these complexes. The results show that the dominant driving force is the enthalpy overcoming an entropic barrier, which is consistent with the conformational selection mechanism, as in the lock-and-key model the interaction is driven by solvent gain in entropy [33-35]. Taking together these observations, chemical shift perturbation, dynamics data and results published by Dobrovolska et al., we argue that the flexible I921-Q923 region of CW is able to make contacts only when complexed with $\mathrm{H} 3 \mathrm{~K} 4 \mathrm{me} 1$ peptide, remaining mobile in other cases, and by that it is involved in selectivity towards $\mathrm{H} 3 \mathrm{~K} 4$ modification.

Conformational analysis with NMR chemical shifts perturbation and thermal stability measurements showed that the conformation of the domain differs when interacting with different methylation states. Dynamic data showed that the structure of $\mathrm{CW}$ is adjusted to the correctly bound form by restricting the mobility of the flexible $\eta 1, \eta 2$, $\eta 3$-loops and I921-Q923 unstructured region. Interaction with the $\mathrm{H} 3 \mathrm{~K} 4 \mathrm{me} 3$ peptide perturbed the conformation to a greater extent than the lower methylation states of peptides, and reduced fold stability markedly. These observations are in agreement with the model presented by Liu et al., where steric hindrances between the binding pocket and trimethylated group were discussed. However, the impact of this steric hindrance is not restricted to the binding site, but appears to affect the whole fold. 

its structural stability through its $\alpha 1$-helix

Compared to other known structures of CW-domains, the ASHH2 subtype has the a1-helix, whose function seems to be related to the specificity of the domains [23]. We show that truncation of the helix eliminates binding by disruption of the domain fold. Point mutations of the amino acids whose side chains are oriented towards the tryptophan binding pocket confirmed that the structure is stabilized by these residues. Comparing the I915A and L919A mutations, we conclude that I915 contributes the most to structural stabilization and is also crucial for maintaining the fold optimal for binding. The I915A mutation had a pronounced effect on the overall structure and chemical environment around the tryptophan side chains of the binding pocket, and thus, likely affected its geometry, which resulted in loss of quantifiable interaction. It was not possible to determine the $T_{m}$ for the 1915A mutant with intrinsic tryptophan fluorescence spectroscopy, as the data could not be fitted by a sigmoidal curve. Knowing from HSQC fingerprinting that the I915A mutant remains folded, such behaviour can be explained by the exposure of tryptophans to the environment [36]. One possibility is that the I915A mutation could distort or displace the $\alpha 1$-helix exposing the tryptophan side chains, as also seems from the change in chemical environment around indole rings of the tryptophans in the HSQC side chain analysis. The L919A mutation showed decreased affinity to H3K4me1 arising from changes in the balance between enthalpy and entropy contributions of the interaction. Higher values for enthalpy and entropy indicate that the binding of the peptide was not complete relative to the native situation, and thermal stability measurements indicated a notable destabilization of the complex. These observations are in agreement with conclusions from Liu et al., where they demonstrated the importance of 1915 and L919 in the formation of the binding pocket. However, their ITC results and conclusion about selectivity of the domain have to be treated with care, as their reported stoichiometric coefficient values did not approach 1:1 binding, as would be expected for a well-optimized system. This deviation might also indicate a low quality sample [37].

To explore the hypothesis that the specificity of the domain might be related to the flexibility of the binding pocket, mutants with variable inter- $\beta$-strand loop were prepared and analyzed. These mutations did not change the binding preference, but the composition and length of the loop between $\beta$ strands did have an impact on binding affinity. The CW-Z1loop mutant also had a significant effect on the fold of the domain. The CW-M3loop mutation did not affect the chemical environment of the tryptophans in the binding pocket, and as the result, the interaction of the CW-M3loop mutant with histone mimicking peptides similar to the WT. The interacation is driven by enthalpy, but with slightly reduced affinities towards $\mathrm{H} 3 \mathrm{~K} 4 \mathrm{me} 2$ and $\mathrm{H} 3 \mathrm{~K} 4 \mathrm{me} 3$. In the case of the $\mathrm{CW}-\mathrm{Z} 1$ loop mutant, the interaction was significantly weakened, and it was not possible to find the biding isotherm by ITC. This loss in affinity can be explained by a high degree of structural perturbance of the domain, as indicated by its HSQC fingerprint.

Ligand binding has different effects on the protein stability depending on the interaction system. Typically, a ligand stabilizes the structure, but in case of binding to partially unfolded intermediate states of proteins, the stability can be decreased [38-41]. Intrinsically disordered proteins interacting with 
ligands are characterized by the formation of secondary structure elements upon binding $[42,43]$. This is, in turn, associated with a reduction in dynamics of flexible loops, shrinking in protein hydrodynamic size and reduction in conformational entropy [41,44-46]. The affinity of the interaction arises from enthalpy-entropy compensations, where the most stable complex is formed with the most favorable thermodynamic terms [47]. With this in mind, our observations indicate that the unique specificity of the ASHH2 CW domain towards $\mathrm{H} 3 \mathrm{~K} 4 \mathrm{me} 1$ modification is coupled with the stabilization of its fold. The stability itself is maintained by the $\alpha 1$-helix via the 1915 and L919 residues, and increases upon complexation with the monomethylated ligand. Consolidation also involves the $\eta 1, \eta 3$-loops and $\mathrm{C}$ terminal coil, and possibly also $\beta$-sheet induction. This consolidation is incomplete and therefore energetically unfavorable when the domain interacts with $\mathrm{H} 3 \mathrm{~K} 4 \mathrm{me} 2$ and $\mathrm{H} 3 \mathrm{~K} 4 \mathrm{me} 3$ peptides.

There is unfortunately little structural information available on how the $\mathrm{CW}$ domain might act in the context of the full ASSH2 protein. It is possible that selectivity towards histones are increased in the full context of the enzyme, or that the $\mathrm{CW}$ domain elicits changes to the domain organization of the full protein when bound. The work done by Dong et al. and $\mathrm{Xu}$ et al. provide some interesting findings regarding this [21,22]. These groups performed methyltransferase assays with a radiography labeled methyl group donor. Xu et al. showed that a truncated construct of ASHH2 lacking the $\mathrm{CW}$ domain and retaining only its catalytically relevant methyltransferase domains, is able to methylate in vitro $\mathrm{H} 3$ histone as well as H4. Dong et al. in their study used a full-length ASHH2, which was shown to "write" a methylation mark on $\mathrm{H} 3$ histone only. These observations together immediately suggest that the $\mathrm{CW}$ domain of ASHH2 methyltransferase might function by restricting the SET domain activity specifically towards $\mathrm{H} 3$ histones.

\section{Materials and methods}

Materials: The Histone $\mathrm{H} 3$ tail mimicking peptides were synthetized by Lifetein (sequences are listed in Table S2) and had 95\% purity (assessed by mass spectrometry). Buffer components and chemicals were purchased from Sigma-Aldrich. $\mathrm{D}_{2} \mathrm{O},{ }^{15} \mathrm{~N}$ enriched $(99 \%) \mathrm{NH}_{4} \mathrm{Cl}$ and ${ }^{13} \mathrm{C}$ enriched (99\%) glucose were purchased from Cambridge Isotopes Laboratories, Inc. (Tewksbury, MA, USA), and SVCP-Super-3-103.5 NMR tubes were acquired from Norell Inc. (Morganton, NC, USA).

Analysis of known structures: To determine the angles between the tryptophan residues in different $\mathrm{CW}$ domains, a multiple structure alignment was performed on the A chain of known $\mathrm{CW}$ domain structures using the POSA web tool [48]. Results were visualized using UCSF Chimera [49] and PyMOL, and the measurements of the angles between the two tryptophan residues were measured manually with Screen Scales from Talon-Designs LLP, and automatically by fitting a plane through the coordinates of the atoms in the two tryptophan side chains and solving the angle between the two planes. Git code is available at https://github.com/oodegard/CW_domain_paper. Sequence alignment was performed using Jalview software [50] with Clustal O algorithm with default parameters. 

its structural stability through its $\alpha 1$-helix

Protein expression and purification: Protein expression and purification was performed as described in [27]. Mutant versions of ASHH2 CW-domain were generated using site-directed mutagenesis and PCR. The primers used are listed in Table S1. After PCR, the reaction mix was treated with DpnI, and linearized DNA was ligated at room temperature for 20 minutes in reaction containing PCR product, NEB4 buffer, T4 ligase, PNK T4 Kinase, 1 mM ATP and 10 mM DTT. Mutant CWconstructs were expressed and purified as described above. All constructs were verified by sequencing.

Isothermal titration calorimetry (ITC): For all measurements, the temperature and stirring rate were kept constant at $25^{\circ} \mathrm{C}$ and $300 \mathrm{rpm}$, respectively. Sample concentration was $50-180 \mu \mathrm{M}$ and the enthalpy of binding was determined by stepwise titration of 400-1800 $\mu \mathrm{M}$ histone peptide. Each CW sample (wt and mutants) were analyzed with H3K4me1, H3K4me2, H3K4me3 (Peptide sequences listed in Table S2). For each experiment, $2 \mu \mathrm{L}$ of peptide was injected 22 times with 300 seconds intervals between injections. Experiments were performed in triplicates. Both protein and peptide were dissolved in $\mathrm{T} 7$ buffer (25 mM Tris-HCL pH 7.0, $150 \mathrm{mM} \mathrm{NaCl}, 1 \mathrm{mM}$ TCEP) and the heat of peptide dilution into T7 buffer was subtracted from the measurement. Binding parameters were determined from the integrated peak areas with independent modelling, using the TA Instruments NanoAnalyze V 2.4.1 software.

Thermal denaturation: The melting point of the different $\mathrm{CW}$ domain constructs, defined as the temperature at the inflection point of I328/I352 during heat denaturation, was determined with and without ligand. Each sample was subjected to a temperature gradient ranging from 5 to $90{ }^{\circ} \mathrm{C}$. Each 5 ${ }^{\circ} \mathrm{C}-10{ }^{\circ} \mathrm{C}$ increase in temperature was allowed to come to thermal equilibrium before measurements were taken. Fluorescence was recorded in the range between 310-410 nm with a scanning rate of 100 $\mathrm{nm} / \mathrm{min}, 2$ scans per sample. Each sample was analyzed in triplicates. The cuvette was equipped with a lid to prevent evaporation from the sample. Samples were dissolved in T7 buffer, protein concentration was 10-20 $\mu \mathrm{M}$ and ratio of protein to peptide was 1:6. Corresponding blanks (buffer or peptide solution) were subtracted from the measurements. Ratio of fluorescence intensities at $328 \mathrm{~nm}$ and $352 \mathrm{~nm}$ were plotted against temperature. A sigmoidal curve with four variables (Equation 1) was fitted between parallels to obtain the inflection point (taken as the melting temperature, $\mathrm{T}_{\mathrm{m}}$ ). Curve fitting was performed with SigmaPlot v13.0 followed by one-way ANOVA statistical test with p-value threshold of 0.05 .

$$
f(x)=y_{0}+\frac{a}{1+e^{\left(-b\left(x-x_{0}\right)\right.}} \quad \text { (Equation 1) }
$$

Here, $f(x)$ is a function of temperature, $y_{0}$ is the slope of the pre-transition state, $a$ is the slope of post-transition state, $x_{0}$ is the infection point of the curve, and $b$ is the slope of the transition state at the inflection point $x_{0}$.

NMR spectroscopy: Protein samples were dissolved in NMR buffer (20 mM Phosphate buffer pH 6.4, $50 \mathrm{mM} \mathrm{NaCl}, 1 \mathrm{mM}$ DTT) with $10 \% \mathrm{D}_{2} \mathrm{O}$. All NMR data was acquired on a Bruker Ascend 850 $\mathrm{MHz}$ instrument, fitted with a cryogenically cooled TCI probe. Data was processed in TopSpin $3.5 \mathrm{pl}$ 

its structural stability through its $\alpha 1$-helix

6.2. ${ }^{1} \mathrm{H}^{15} \mathrm{~N}-\mathrm{HSQC}$ fingerprints were acquired using the $\mathrm{f} 3$ channel, ${ }^{15} \mathrm{~N}$ decoupling during acquisition, water flip-back pulse and echo/anti-echo-TPPI gradient selection [51]. Typical parameters were: p1 7.06

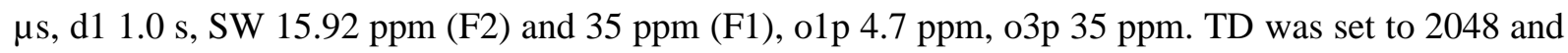
128 in F2 and F1, respectively. Data was acquired using 50\% non-uniform sampling. For processing, a forward complex linear prediction was applied in F1 up to 256 points and zero-filled up to 512 points. The FIDs were apodized using a squared cosine function in both dimensions prior to Fouriertransformation. HNCA, HNcoCA, CBCAcoNH and CBCANH spectra were acquired for assignment purposes. All experiments were acquired using time-optimized NMR [52], and were set up using the standard parameter files provided by the software of the instrument provider. Typical acquisition and processing parameters were as above, except that TD for the ${ }^{1} \mathrm{H}$-dimension was 698 and SW was 14.00 $\mathrm{ppm}$. In addition, the TD for ${ }^{13} \mathrm{C}$-dimension was (where applicable) 64 points with linear prediction up to 80 . NUS-amount for the 3D experiments was set to $25 \%$. Processed NMR data was imported into CARA [53], for assignment and further analysis. Assigned HSQC spectra for wild type CW in unbound state and bound to corresponding peptides were used to calculate chemical shift perturbations with equation 2 and scaling factor $\alpha_{N}$ of $0.17[54,55]$.

$$
\Delta p p m=\sqrt{\left(\Delta \delta_{H N}\right)^{2}+\left(\Delta \delta_{N} * \alpha_{N}\right)^{2}} \quad(\text { Equation 2) }
$$

Here, $\Delta p p m$ is combined chemical shift; $\Delta \delta_{H N}$ is amide proton chemical shift, ppm; $\Delta \delta_{N}$ is nitrogen chemical shift, ppm; $\alpha_{N}$ is scaling factor.

Dynamics measurement: Dynamics measurements were performed as described in [24]. Briefly, for determination of heteronuclear NOEs, two ${ }^{1} \mathrm{H}^{-15} \mathrm{~N}$ HSQC data sets were recorded. A recycling delay of $3 \mathrm{~s}$ was used between transients. Relaxation delays of 20,60, 80, 100, 200, 400, 600, $800,1000,1200$, and $1400 \mathrm{~ms}$ were recorded for T1 measurements, and relaxation delays of 16, 30, 60, $95,125,160,190,220,250,345,440$, and $500 \mathrm{~ms}$ were recorded for $\mathrm{T} 2$ measurements. The heteronuclear NOE values were calculated as the ratio of the steady-state intensities measured with and without saturation of the proton magnetization. Data analysis was done in Bruker Dynamics Center 2.5.3 (Bruker BioSpin).

\section{Acknowledgements}

The work was supported through the Norwegian NMR Platform, NNP (grant 226244 / F50) in the form of instruments and experimental time. The authors wish to thank the NNP Bergen Node for facilitating the research. We would also like to thank Carol Issalene for help with protein purification, Jean-Karim Hériché (European Molecular Biology Laboratory) for help with the calculation of tryptophan angles, and Morten Andreas Govasli Larsen for the help with mutagenesis.

\section{Conflict of interests}

Authors declare no conflict of interests. 


\section{Author contributions}

Designed experiments: MB, ØH, ØØF, ØS. Performed experiments: MB, ØH. Analyzed data and interpreted results: MB, OD, ØН, ØØF, RAA, ØS. Wrote drafts: MB, ØН. Revised drafts: MB, ØН, OD, ØS. Prepared Figures: MB, OD, ØH. Data curation: ØH, OD. Formulated research questions and wrote grants: RAA, ØH.

\section{References}

[1] Teske, K.A. and Hadden, M.K. (2017). Methyllysine binding domains: Structural insight and small molecule probe development. Eur J Med Chem 136, 14-35.

[2] Patel, D.J. (2016). A Structural Perspective on Readout of Epigenetic Histone and DNA Methylation Marks. Cold Spring Harb Perspect Biol 8, a018754.

[3] DesJarlais, R. and Tummino, P.J. (2016). Role of Histone-Modifying Enzymes and Their Complexes in Regulation of Chromatin Biology. Biochemistry 55, 1584-99.

[4] Taverna, S.D., Li, H., Ruthenburg, A.J., Allis, C.D. and Patel, D.J. (2007). How chromatinbinding modules interpret histone modifications: lessons from professional pocket pickers. Nat Struct Mol Biol 14, 1025-1040.

[5] Sanchez, R. and Zhou, M.M. (2011). The PHD finger: a versatile epigenome reader. Trends Biochem Sci 36, 364-72.

[6] Mellor, J. (2006). It takes a PHD to read the histone code. Cell 126, 22-4.

[7] Perry, J. and Zhao, Y. (2003). The CW domain, a structural module shared amongst vertebrates, vertebrate-infecting parasites and higher plants. Trends Biochem Sci 28, 576-80.

[8] He, F. et al. (2010). Structural insight into the zinc finger CW domain as a histone modification reader. Structure 18, 1127-39.

[9] Hoppmann, V., Thorstensen, T., Kristiansen, P.E., Veiseth, S.V., Rahman, M.A., Finne, K., Aalen, R.B. and Aasland, R. (2011). The CW domain, a new histone recognition module in chromatin proteins. EMBO J 30, 1939-52.

[10] Andrews, F.H. et al. (2016). Multivalent Chromatin Engagement and Inter-domain Crosstalk Regulate MORC3 ATPase. Cell Rep 16, 3195-3207.

[11] Li, S. et al. (2016). Mouse MORC3 is a GHKL ATPase that localizes to H3K4me3 marked chromatin. Proc Natl Acad Sci U S A 113, E5108-16.

[12] Zhang, Y. et al. (2019). Mechanism for autoinhibition and activation of the MORC3 ATPase. Proc Natl Acad Sci U S A

[13] Tencer, A.H. et al. (2020). Molecular mechanism of the MORC4 ATPase activation. Nat Commun 11, 5466.

[14] Wang, Y., Reddy, B., Thompson, J., Wang, H., Noma, K., Yates, J.R. and Jia, S. (2009). Regulation of Set9-mediated H4K20 methylation by a PWWP domain protein. Mol Cell 33, 428-37.

[15] Liu, Y., Tempel, W., Zhang, Q., Liang, X., Loppnau, P., Qin, S. and Min, J. (2016). Familywide Characterization of Histone Binding Abilities of Human CW Domain-containing Proteins. J Biol Chem 291, 9000-13.

[16] Zhang, Q. et al. (2013). Structure-function analysis reveals a novel mechanism for regulation of histone demethylase LSD2/AOF1/KDM1b. Cell Res 23, 225-41.

[17] Yang, Z. et al. (2010). AOF1 is a histone H3K4 demethylase possessing demethylase activityindependent repression function. Cell Res 20, 276-87.

[18] Shi, Y., Lan, F., Matson, C., Mulligan, P., Whetstine, J.R., Cole, P.A. and Casero, R.A. (2004). Histone demethylation mediated by the nuclear amine oxidase homolog LSD1. Cell 119, 94153.

[19] Questa, J.I., Rius, S.P., Casadevall, R. and Casati, P. (2016). ZmMBD101 is a DNA-binding protein that maintains Mutator elements chromatin in a repressive state in maize. Plant Cell Environ 39, 174-84. 
[20] Grini, P.E., Thorstensen, T., Alm, V., Vizcay-Barrena, G., Windju, S.S., Jørstad, T.S., Wilson, Z.A. and Aalen, R.B. (2009). The ASH1 HOMOLOG 2 (ASHH2) histone H3 methyltransferase is required for ovule and anther development in Arabidopsis. PLoS One 4, e7817.

[21] Dong, G., Ma, D.P. and Li, J. (2008). The histone methyltransferase SDG8 regulates shoot branching in Arabidopsis. Biochem Biophys Res Commun 373, 659-64.

[22] Xu, L., Zhao, Z., Dong, A., Soubigou-Taconnat, L., Renou, J.P., Steinmetz, A. and Shen, W.H. (2008). Di- and tri- but not monomethylation on histone H3 lysine 36 marks active transcription of genes involved in flowering time regulation and other processes in Arabidopsis thaliana. Mol Cell Biol 28, 1348-60.

[23] Liu, Y. and Huang, Y. (2018). Uncovering the mechanistic basis for specific recognition of monomethylated $\mathrm{H} 3 \mathrm{~K} 4$ by the $\mathrm{CW}$ domain of Arabidopsis histone methyltransferase SDG8. J Biol Chem

[24] Dobrovolska, O. et al. (2020). The Arabidopsis (ASHH2) CW domain binds monomethylated $\mathrm{K} 4$ of the histone $\mathrm{H} 3$ tail through conformational selection. FEBS J

[25] Csermely, P., Palotai, R. and Nussinov, R. (2010). Induced fit, conformational selection and independent dynamic segments: an extended view of binding events. Trends Biochem Sci 35, $539-46$.

[26] Tsai, C.J., Kumar, S., Ma, B. and Nussinov, R. (1999). Folding funnels, binding funnels, and protein function. Protein Sci 8, 1181-90.

[27] Dobrovolska, O., Bril'kov, M., Ødegård-Fougner, Ø., Aasland, R. and Halskau, Ø. (2018). 1H, $13 \mathrm{C}$, and $15 \mathrm{~N}$ resonance assignments of $\mathrm{CW}$ domain of the N-methyltransferase ASHH2 free and bound to the mono-, di- and tri-methylated histone H3 tail peptides. Biomolecular NMR Assignments

[28] Berggård, T., Linse, S. and James, P. (2007). Methods for the detection and analysis of proteinprotein interactions. Proteomics 7, 2833-42.

[29] Schuck, P. and Zhao, H. (2010). The role of mass transport limitation and surface heterogeneity in the biophysical characterization of macromolecular binding processes by SPR biosensing. Methods Mol Biol 627, 15-54.

[30] Nguyen, H.H., Park, J., Kang, S. and Kim, M. (2015). Surface plasmon resonance: a versatile technique for biosensor applications. Sensors (Basel) 15, 10481-510.

[31] Ladbury, J.E. (2010). Calorimetry as a tool for understanding biomolecular interactions and an aid to drug design. Biochem Soc Trans 38, 888-93.

[32] Homans, S.W. (2007) Dynamics and Thermodynamics of Ligand-Protein Interactions. In Bioactive Conformation I (Peters, T., ed.^eds), pp. 51-82. Springer Berlin Heidelberg, Berlin, Heidelberg.

[33] Du, X., Li, Y., Xia, Y.L., Ai, S.M., Liang, J., Sang, P., Ji, X.L. and Liu, S.Q. (2016). Insights into Protein-Ligand Interactions: Mechanisms, Models, and Methods. Int J Mol Sci 17

[34] Corbett, P.T., Tong, L.H., Sanders, J.K. and Otto, S. (2005). Diastereoselective amplification of an induced-fit receptor from a dynamic combinatorial library. J Am Chem Soc 127, 8902-3.

[35] Li, H., Xie, Y., Liu, C. and Liu, S. (2014). Physicochemical bases for protein folding, dynamics, and protein-ligand binding. Sci China Life Sci 57, 287-302.

[36] Duy, C. and Fitter, J. (2006). How aggregation and conformational scrambling of unfolded states govern fluorescence emission spectra. Biophys J 90, 3704-11.

[37] Perozzo, R., Folkers, G. and Scapozza, L. (2004). Thermodynamics of protein-ligand interactions: history, presence, and future aspects. J Recept Signal Transduct Res 24, 1-52.

[38] Matulis, D., Kranz, J.K., Salemme, F.R. and Todd, M.J. (2005). Thermodynamic stability of carbonic anhydrase: measurements of binding affinity and stoichiometry using ThermoFluor. Biochemistry 44, 5258-66.

[39] Cimmperman, P. et al. (2008). A quantitative model of thermal stabilization and destabilization of proteins by ligands. Biophys J 95, 3222-31.

[40] Gianni, S. and Jemth, P. (2019). Affinity versus specificity in coupled binding and folding reactions. Protein Eng Des Sel 32, 355-357.

[41] D'Auria, S. et al. (2005). Binding of glutamine to glutamine-binding protein from Escherichia coli induces changes in protein structure and increases protein stability. Proteins 58, 80-7. 
[42] Schneider, R., Maurin, D., Communie, G., Kragelj, J., Hansen, D.F., Ruigrok, R.W., Jensen, M.R. and Blackledge, M. (2015). Visualizing the molecular recognition trajectory of an intrinsically disordered protein using multinuclear relaxation dispersion NMR. J Am Chem Soc $137,1220-9$.

[43] Iešmantavičius, V., Dogan, J., Jemth, P., Teilum, K. and Kjaergaard, M. (2014). Helical propensity in an intrinsically disordered protein accelerates ligand binding. Angew Chem Int Ed Engl 53, 1548-51.

[44] Zhu, J., Qi, R., Liu, Y., Zhao, L. and Han, W. (2019). Mechanistic Insights into the Effect of Ligands on Structural Stability and Selectivity of Sulfotransferase 2A1 (SULT2A1). ACS Omega 4, 22021-22034.

[45] Di Lella, S., Martí, M.A., Croci, D.O., Guardia, C.M., Díaz-Ricci, J.C., Rabinovich, G.A., Caramelo, J.J. and Estrin, D.A. (2010). Linking the structure and thermal stability of betagalactoside-binding protein galectin-1 to ligand binding and dimerization equilibria. Biochemistry 49, 7652-8.

[46] Steiner, S., Magno, A., Huang, D. and Caflisch, A. (2013). Does bromodomain flexibility influence histone recognition? FEBS Lett 587, 2158-63.

[47] Ferrante, A. and Gorski, J. (2012). Enthalpy-entropy compensation and cooperativity as thermodynamic epiphenomena of structural flexibility in ligand-receptor interactions. J Mol Biol 417, 454-67.

[48] Ye, Y. and Godzik, A. (2004). FATCAT: a web server for flexible structure comparison and structure similarity searching. Nucleic Acids Res 32, W582-5.

[49] Pettersen, E.F., Goddard, T.D., Huang, C.C., Couch, G.S., Greenblatt, D.M., Meng, E.C. and Ferrin, T.E. (2004). UCSF Chimera--a visualization system for exploratory research and analysis. J Comput Chem 25, 1605-12.

[50] Waterhouse, A.M., Procter, J.B., Martin, D.M., Clamp, M. and Barton, G.J. (2009). Jalview Version 2--a multiple sequence alignment editor and analysis workbench. Bioinformatics 25, 1189-91.

[51] Schleucher, J., Schwendinger, M., Sattler, M., Schmidt, P., Schedletzky, O., Glaser, S.J., Sørensen, O.W. and Griesinger, C. (1994). A general enhancement scheme in heteronuclear multidimensional NMR employing pulsed field gradients. J Biomol NMR 4, 301-6.

[52] Lescop, E., Schanda, P. and Brutscher, B. (2007). A set of BEST triple-resonance experiments for time-optimized protein resonance assignment. J Magn Reson 187, 163-9.

[53] Keller, R.L.J. (2005) Optimizing the process of nuclear magnetic resonance spectrum analysis and computer aided resonance assignmented.^eds)

[54] Tochio, H., Hung, F., Li, M., Bredt, D.S. and Zhang, M. (2000). Solution structure and backbone dynamics of the second PDZ domain of postsynaptic density-95. J Mol Biol 295, 225-37.

[55] Piserchio, A., Pellegrini, M., Mehta, S., Blackman, S.M., Garcia, E.P., Marshall, J. and Mierke, D.F. (2002). The PDZ1 domain of SAP90. Characterization of structure and binding. J Biol Chem 277, 6967-73. 


\section{FIGURES AND TABLES}

\section{Table 1}

Angles between tryptophan side chains forming the binding pocket. Tryptophan angle measurements were performed manually with Screen Scales from Talon-Designs LLP and automatically by fitting a plane through the coordinates of the atoms in the two tryptophan side chains and solving the angle between the two planes.

\begin{tabular}{|c|c|c|c|c|c|c|}
\hline Uniprot ID & PDB ID & Ligand & $\begin{array}{l}\text { Angle, } \\
\text { manual }\end{array}$ & $\begin{array}{c}\text { Angle, } \\
\text { automatic }\end{array}$ & $\begin{array}{c}\text { Highest } \\
\text { specificity }\end{array}$ & Reference \\
\hline \multirow[t]{3}{*}{ ASHH2_ARATH } & 2L7P & Unbound & 65.9 & 64.9 & \multirow[t]{3}{*}{ H3K4me1 } & \multirow[t]{3}{*}[9,23,24]{} \\
\hline & 6QXZ & $\mathrm{H} 3 \mathrm{~K} 4 \mathrm{me} 1$ & 94.4 & 94.3 & & \\
\hline & $5 Y V X$ & $\mathrm{H} 3 \mathrm{~K} 4 \mathrm{me} 1$ & 113.0 & 112.6 & & \\
\hline \multirow{3}{*}{ MORC3_HUMAN } & 4QQ4 & $\mathrm{H} 3 \mathrm{~K} 4$ & 99.6 & 99.7 & \multirow[t]{3}{*}{ H3K4me3 } & \multirow{3}{*}[10,15]{} \\
\hline & $5 S V Y$ & $\mathrm{H} 3 \mathrm{~K} 4 \mathrm{me} 1$ & 99.1 & 98.1 & & \\
\hline & $5 \mathrm{SVX}$ & $\mathrm{H} 3 \mathrm{~K} 4 \mathrm{me} 3$ & 100.5 & 100.1 & & \\
\hline \multirow[t]{2}{*}{ MORC3_MOUSE } & $5 \mathrm{IX} 2$ & $\mathrm{H} 3 \mathrm{~K} 4$ & 78.0 & 76.8 & \multirow[t]{2}{*}{$\mathrm{H} 3 \mathrm{~K} 4 \mathrm{me} 3$} & \multirow[t]{2}{*}[11]{} \\
\hline & $5 \mathrm{IX} 1$ & $\mathrm{H} 3 \mathrm{~K} 4 \mathrm{me} 3$ & 89.9 & 88.6 & & \\
\hline \multirow[t]{2}{*}{ ZCWPW1_HUMAN } & 2E61 & Unbound & 104.1 & 104.3 & \multirow[t]{2}{*}{$\mathrm{H} 3 \mathrm{~K} 4 \mathrm{me} 3$} & \multirow[t]{2}{*}{ [8] } \\
\hline & 2RR4 & $\mathrm{H} 3 \mathrm{~K} 4 \mathrm{me} 3$ & 107.6 & 105.7 & & \\
\hline ZCWPW2_HUMAN & 4062 & $\mathrm{H} 3 \mathrm{~K} 4 \mathrm{me} 3$ & 113.2 & 111.2 & H3K4me3 & {$[15]$} \\
\hline
\end{tabular}


Figure 1
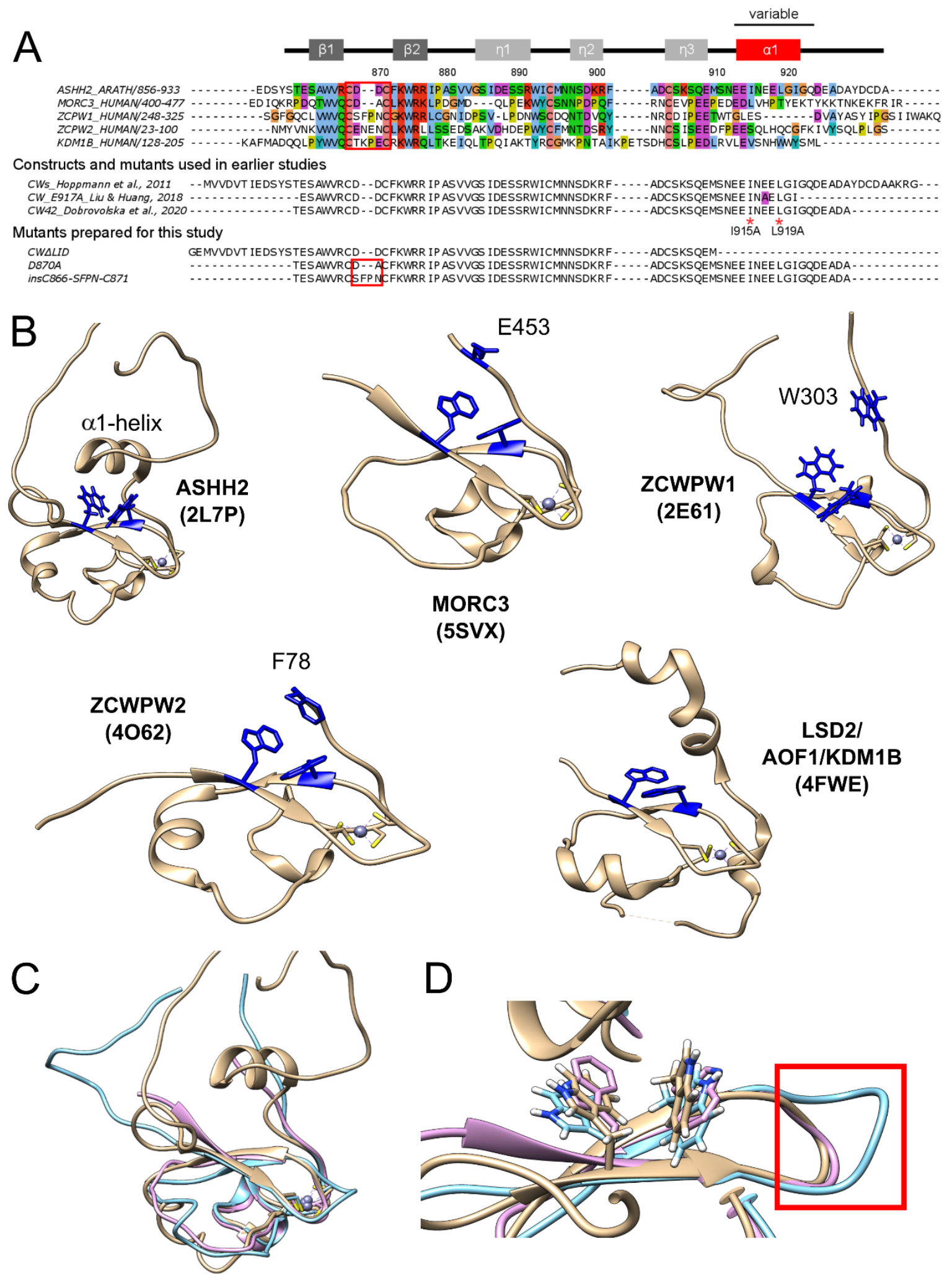

Figure 1: Overview of CW domains and structural analysis. A - TOP: Sequence alignment of CWdomains of ASHH2 methyltransferase (Arabidopsis thaliana, Q2LAE1), MORC3 protein (Homo 
sapiens, Q14149), ZCWPW1 protein (Homo sapiens, Q9H0M4), ZCWPW2 protein (Homo sapiens, Q504Y3) and KDM1B histone demethylase (Homo sapiens, Q08EI0). The alignment was prepared using Jalview software and UniProt entries with ClustalO default parameters, and Clustalx coloring scheme was used. MIDDLE: CW domain constructs used in earlier studies on the ASHH2 CW domain by Hoppmann et al., 2011, Liu and Huang, 2018 and Dobrovolska et al., 2020 [9,23,24]. The E917A mutation used by Liu and Huang, 2018 is marked by purple color. I915A and L919A mutations analyzed by Liu and Huang, 2018 and in this work are marked by red * symbols. BOTTOM: Sequences of the additional mutants (CW LLID, D870A and insC866-SFPN-C871) prepared exclusively for this work. The secondary structure of $\mathrm{CW}$ is indicated at the top of the panel. The red squares indicate the variale loop situated between the $\beta$-strands that forms part of the binding site. These constructs also have a Nterminal sequence ("GSRRASVGSEF"), that is not shown in the figure; B - Overview of CW domain structures: ASHH2 (2L7P), MORC3 (5SXV), ZCWPW1 (2E61), ZCWPW2 (4O62) and LSD2/AOF1/KDM1B (4FWE) (tryptophans forming binding pockets and the variable C-terminal region residues, implicated in binding, are highlighted in blue); $\mathrm{C}$ - superposition of $\mathrm{CW}$ domain structures: ASHH2 (brown), MORC3 (pink), and ZCWPW1 (blue); D - close-up view of binding pocket. The loops between the $\beta$-strands subject to the insC866-SFPN-C871 mutationare indicated by red square. Graphics were prepared using the UCSF Chimera software and the pdb files represents domains in their unbound state. 
Figure 2
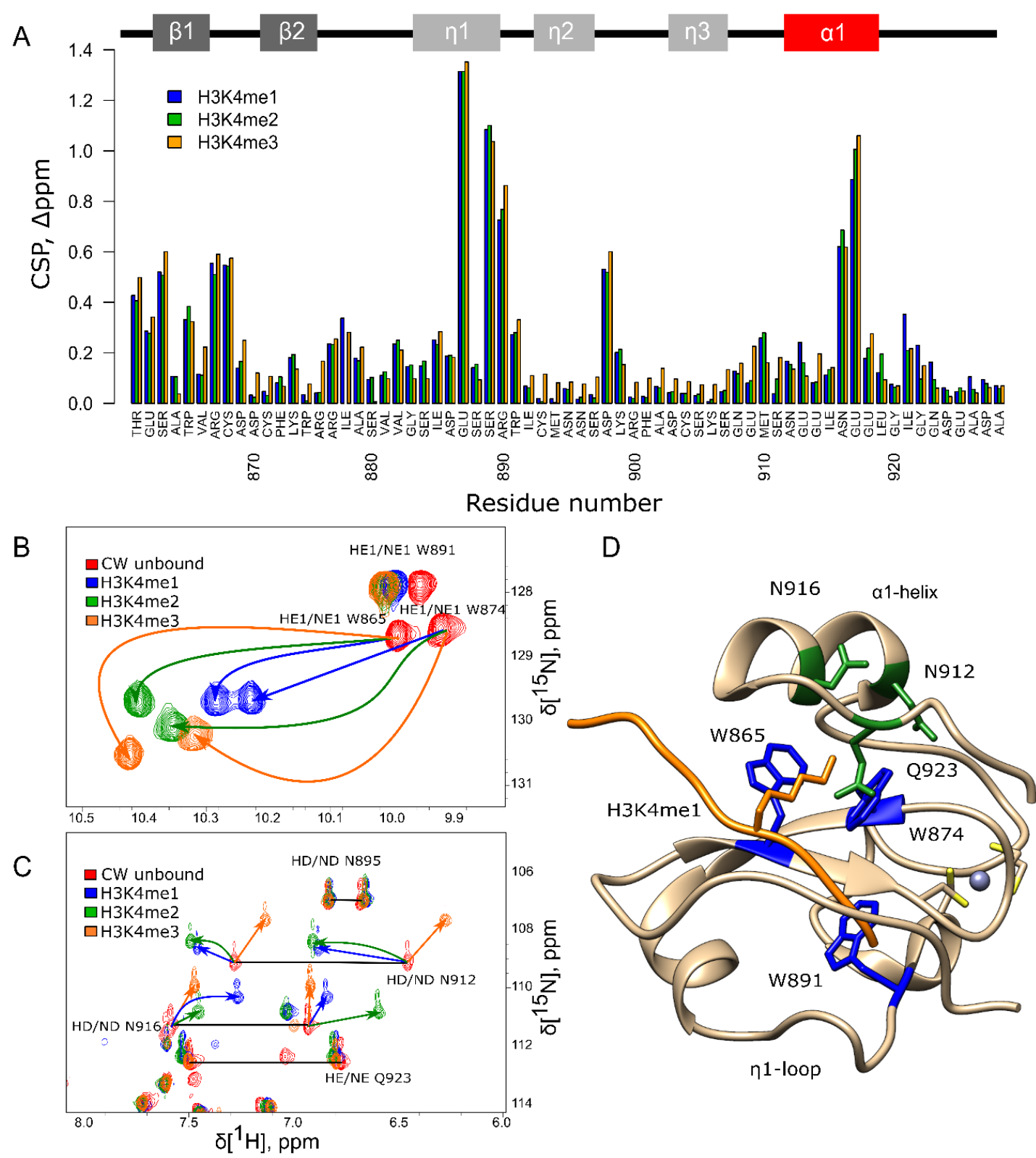

Figure 2: NMR analysis of CW interacting with histone mimicking peptides. $\mathrm{A}$ - chemical shift perturbation, calculated for $\mathrm{CW}$ bound to corresponding peptides (H3K4me1 blue, H3K4me2 green and H3K4me3 orange). Secondary structure of CW is indicated at the top of the panel; B and C - chemical shift of tryptophan, asparagine and glutamine side chains signals under binding of H3K4me1 (blue), H3K4me2 (green) and H3K4me3 (orange) peptides (red color - CW domain in unbound state); horizontal black lines connect signal pairs for ${ }^{15} \mathrm{~N} \varepsilon$ and ${ }^{15} \mathrm{~N} \delta$ signals of glutamines and aspargines respectively; D - side chains tryptophans (highlighted in blue), asparagines and glutamine (highlighted in green) mapped on $\mathrm{CW}$ structure in bound to $\mathrm{H} 3 \mathrm{~K} 4 \mathrm{me} 1$ peptide (orange color) conformation (PDB: 6QXZ); grey sphere is $\mathrm{Zn}^{2+}$ ion coordinated by cysteins (yellow). 
Figure 3
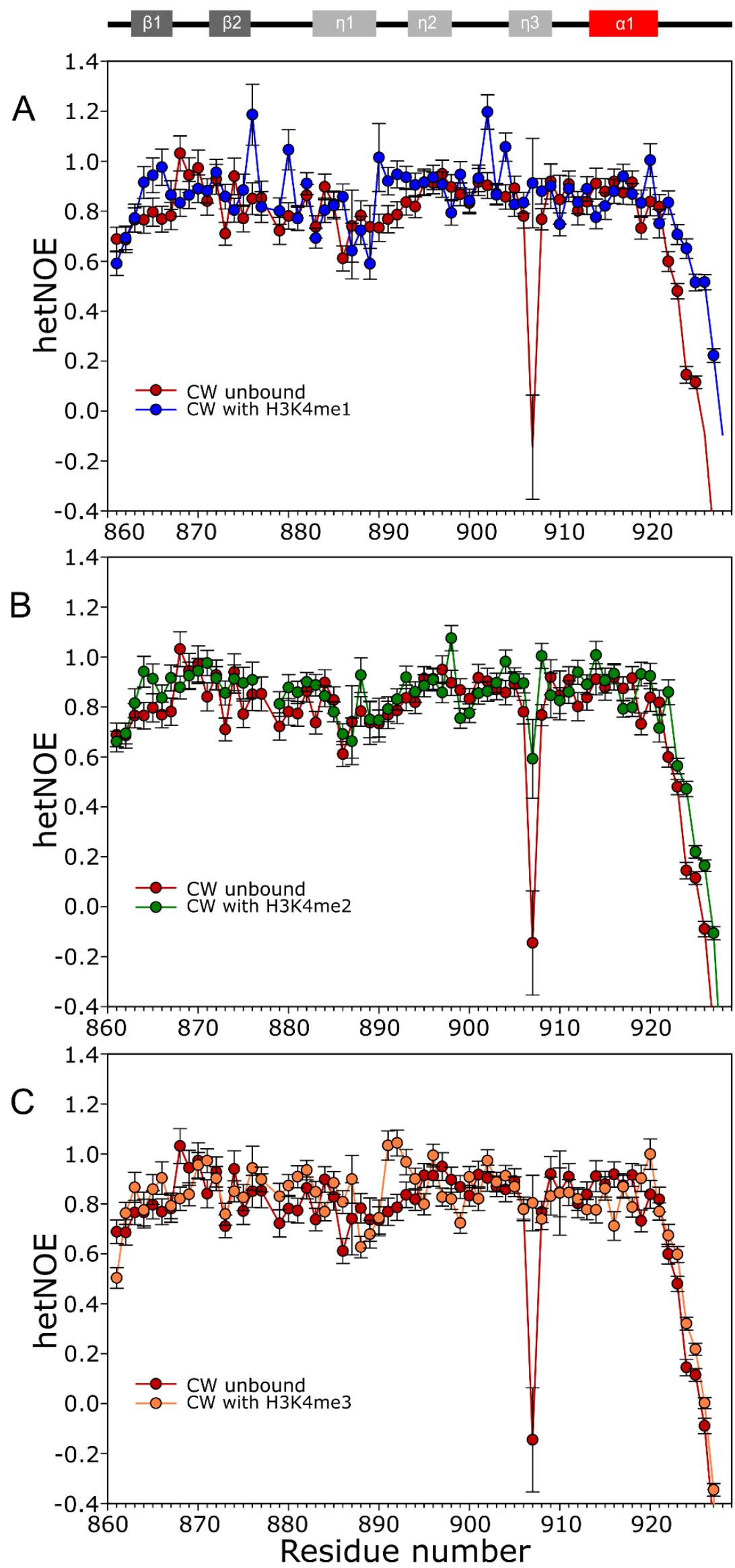
Figure 3: Heteronuclear ${ }^{1} \mathrm{H}-{ }^{15} \mathrm{~N}$ NOEs data of $\mathrm{CW}$ bound to histone mimicking peptides. $\mathrm{A}-\mathrm{CW}$ with H3K4me1 (blue circles, ); B - CW with H3K4me2 peptide (green circles, 0 ); and C - CW with H3K4me3 peptide (orange circles, $)$ ). The data in each panel is overlaid with unbound $\mathrm{CW}$ (red circles, ). Secondary structure of $\mathrm{CW}$ is indicated at the top of the panels.

Figure 4
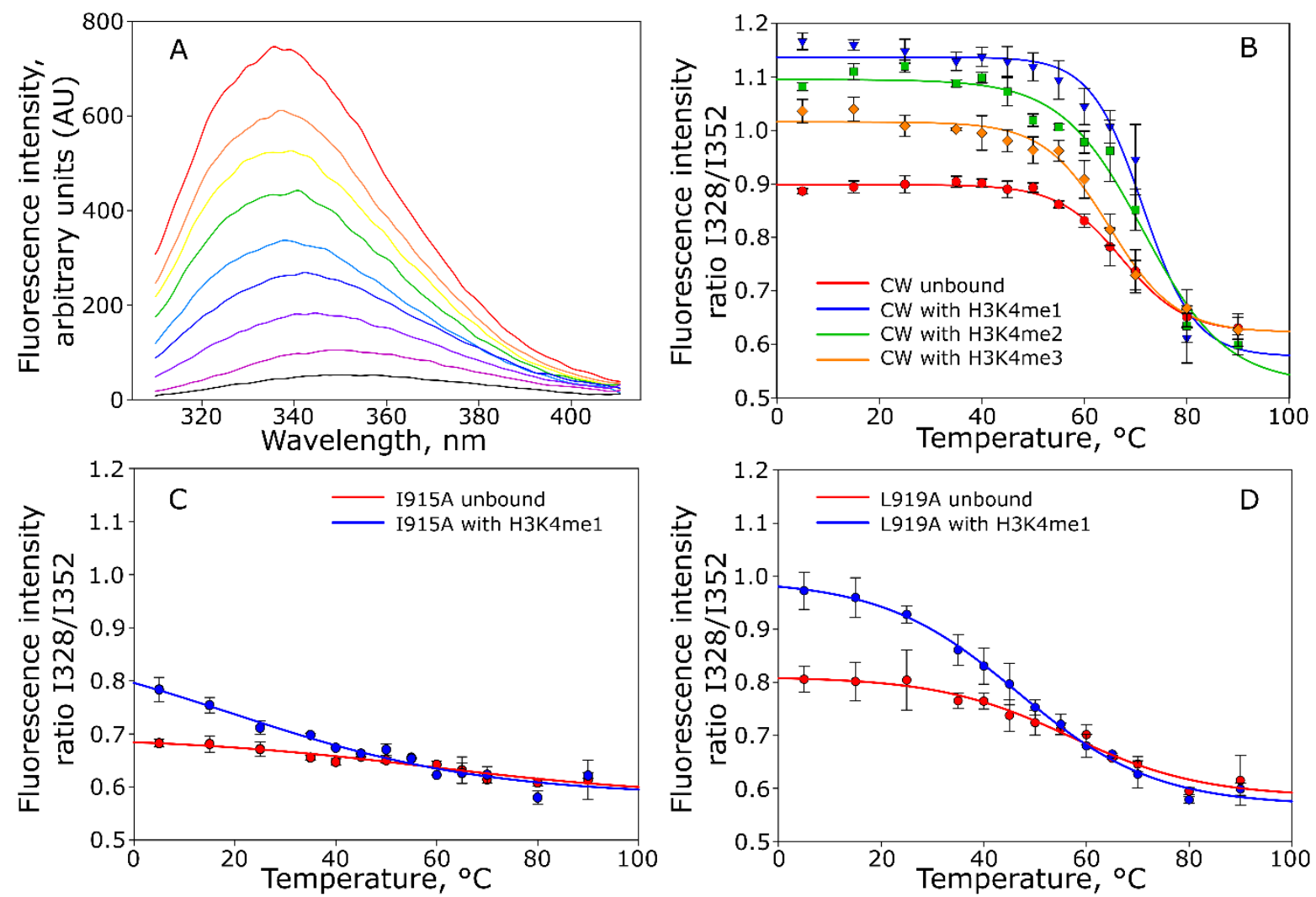

Figure 4: Thermal denaturation of CW monitore by intrinsic tryptophan fluorescence in unbound and bound states. A - Representative fluorescence traces for wild type CW bound to H3K4me1 (only selected traces are shown); B - melting curves for unbound CW domain ( - ) and CW domain in presence of H3K4me1 (-), H3K4me2 (-) and H3K4me3 (-) peptides; C - melting curves for unbound CW I915A mutant (-) and in presence of H3K4me1 peptide (-); D - melting curves for unbound CW L919A mutant (-) and in presence of H3K4me1 peptide (-). Number of replicates $=3$. 
Figure 5
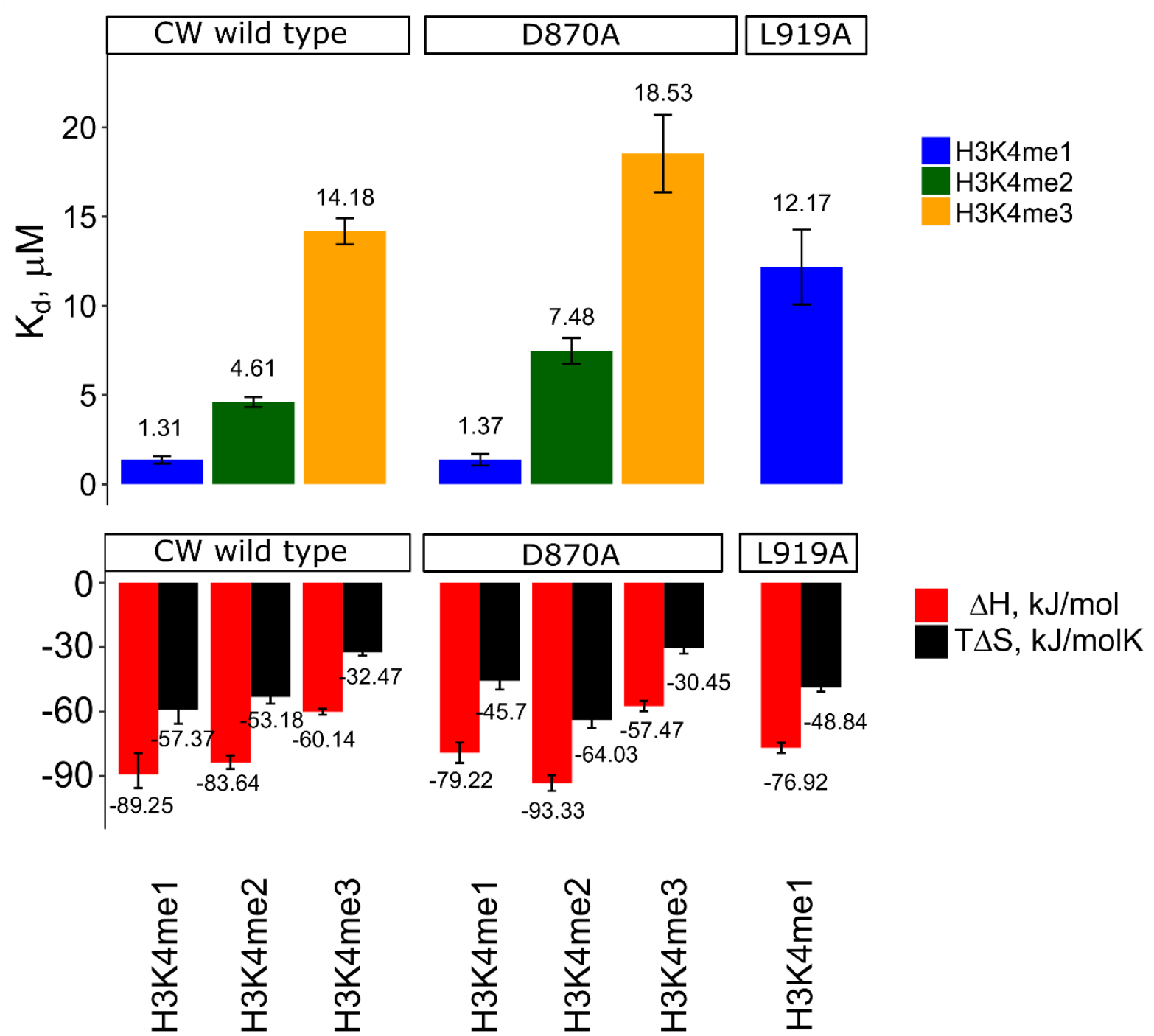

Figure 5: $\mathbf{K}_{\mathrm{d}}, \Delta \mathbf{H}$ and $\mathbf{T} \Delta \mathbf{S}$ values determined by ITC. Data shown only for WT $\mathrm{CW}$ and mutants D870A and L919A, as insC866-SFPN-C871 and I915A did not produced interpretable isotherms. Number of replicates $=3$. Error bars show standard deviation. Representable isotherms can be found in supplementary Fig. S2. 
Figure 6

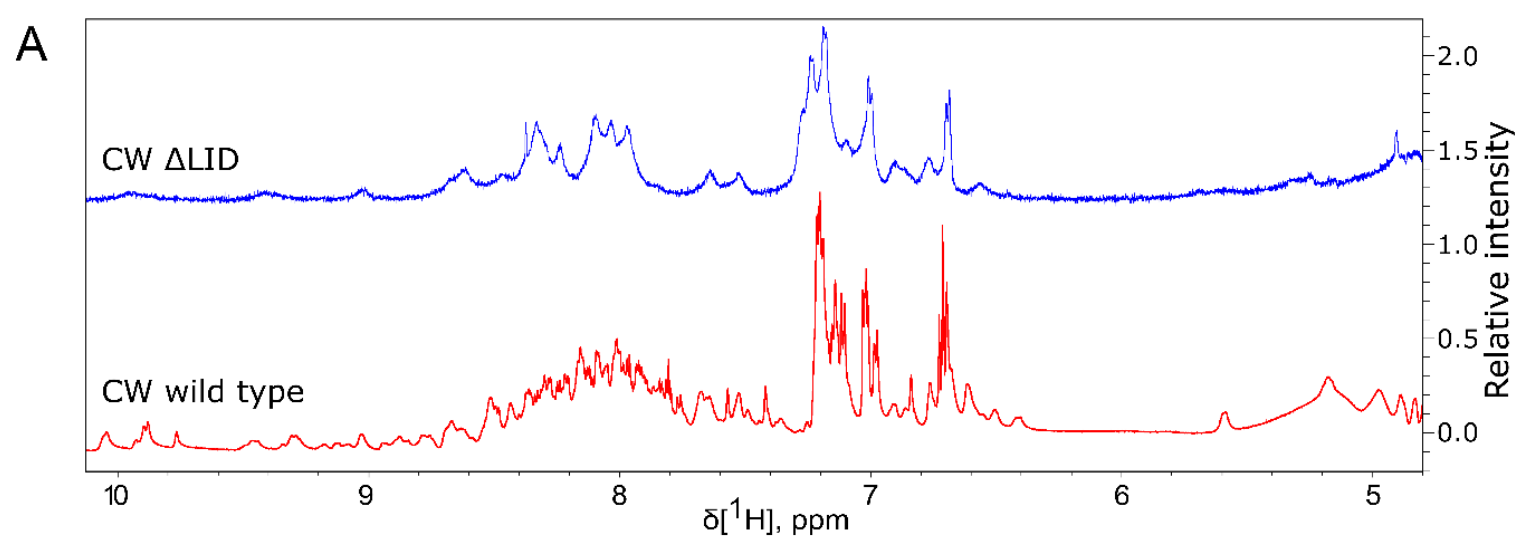

B
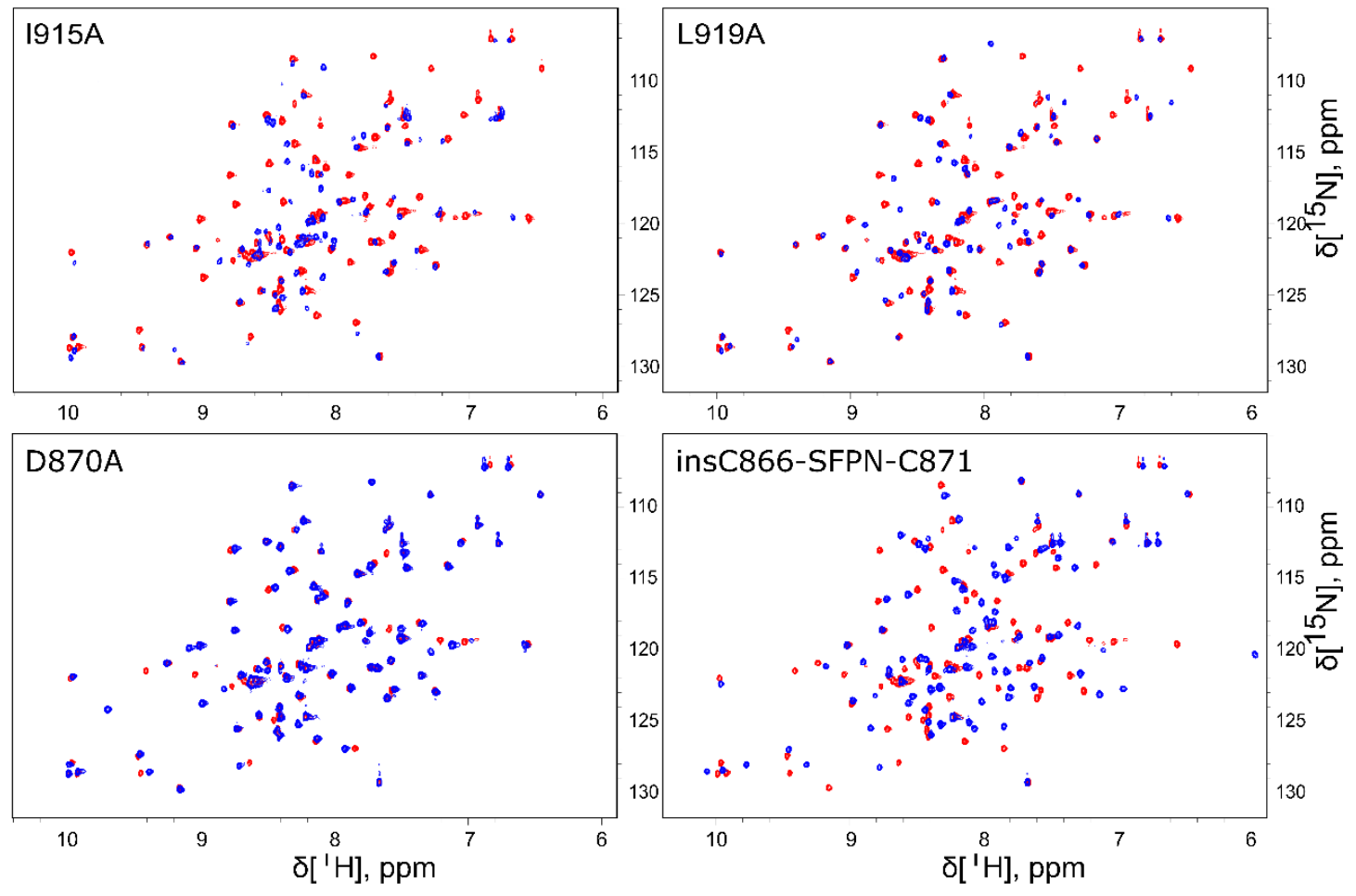

C
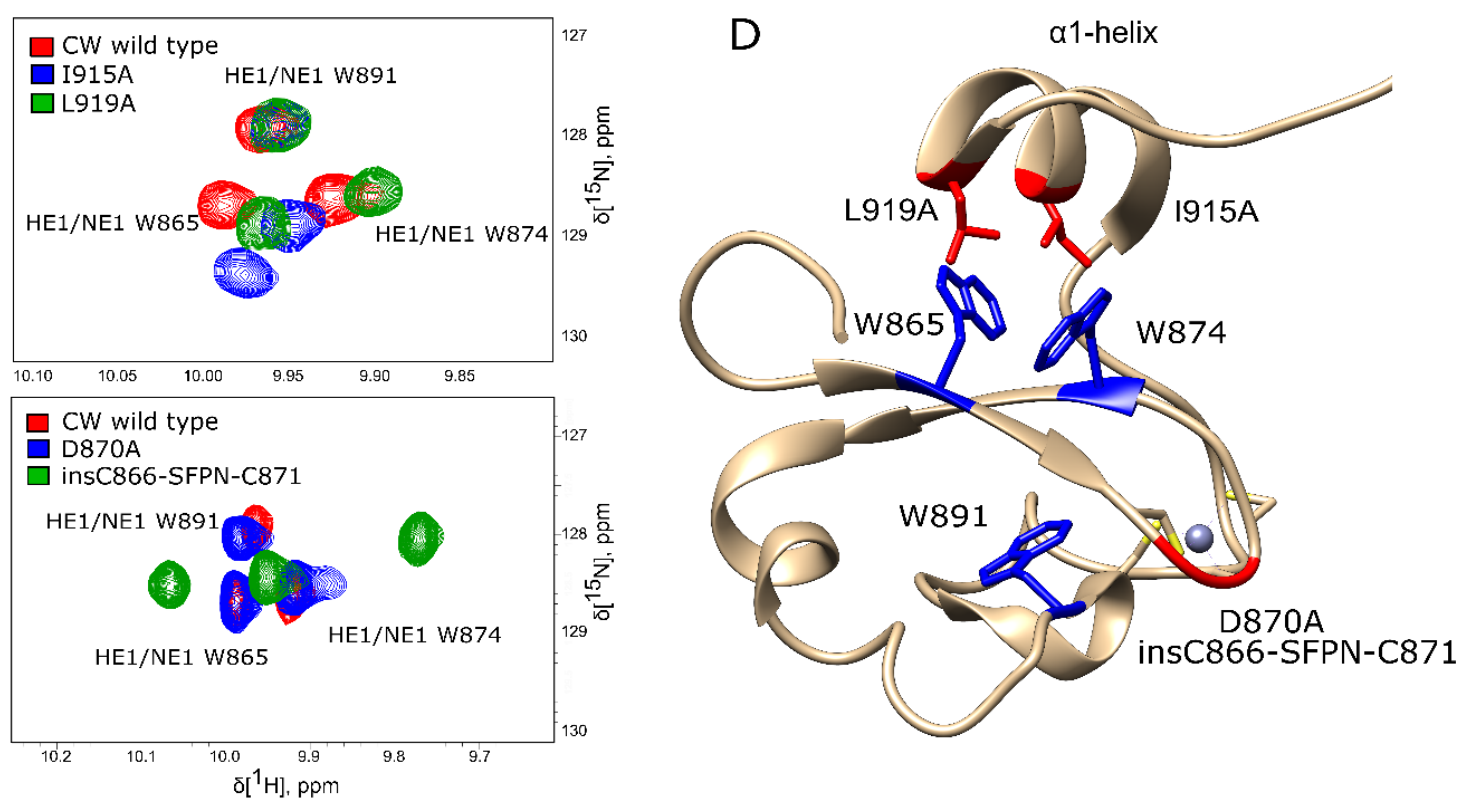

its structural stability through its $\alpha 1$-helix

Figure 6: Mutation induced structural disturbances analyzed by NMR. A - proton spectrum of CW $\triangle$ LID (blue) compared to the proton spectrum of wild type CW (red). The CW $\Delta$ LID trace has been shifted up by 1.5 relative units to make comparison easier. B - ${ }^{15} \mathrm{~N}$ HSQC spectra of I915A, L919A, D870A and insC886-SFPN-C871 (blue color) overlaid with spectra of WT CW (red color). C - the effect of mutations in $\alpha 1$-helix (red - WT CW, blue - I915A, green - L919A (top panel)), and the effect of mutations in the loop between $\beta$-sheets (red - WT CW, blue - D870A, green - insC866-SFPN-C871 (bottom panel)). D - location of the point mutations (red sticks) and insertion mutations (red backbone trace) mapped on the CW structure (PDB: 2L7P). The tryptophans are highlighted in blue; grey sphere is $\mathrm{Zn}^{2+}$ ion coordinated by cysteins (yellow). 